\title{
Insulin Distress
}

\author{
Sanjay Kalra ${ }^{1}$ and Yatan Pal Singh Balhara ${ }^{2}$
}

1. Department of Endocrinology, Bharti Hospital, Karnal, India; 2. Department of Psychiatry, National Drug Dependence Treatment Centre, All India Institute of Medical Sciences, New Delhi, India

$\mathrm{D}$ iabetes distress (DD) is a commonly encountered adjustment disorder among persons living with diabetes. This article discusses insulin distress, a subtype of DD, which has distinct characteristics. Insulin distress can be defined as an emotional response to a suggestion to use insulin; characterized by extreme apprehension, discomfort, dejection, or denial; due to a perceived inability to cope with the requirements of insulin therapy. We describe the etiopathogenesis, clinical features and management of insulin distress. They highlight the clinical challenges that insulin distress poses in some persons. Pragmatic suggestions are shared regarding the addressing of insulin distress before or in parallel with insulin prescription.

DOI: https://doi.org/10.17925/USE.2018.14.1.27

\section{Keywords}

Clinical inertia, insulin, insulin initiation, insulin intensification, psychosocial aspects

Disclosure: Sanjay Kalra and Yatan Pal Singh Balhara have nothing to declare in relation to this article.

Review Process: This article is a short opinion piece and has not been submitted to external peer reviewers.

Authorship: All named authors meet the International Committee of Medical Journal Editors (ICMJE) criteria for authorship of this manuscript, take responsibility for the integrity of the work as a whole, and have given final approval to the version to be published.

open Access: This article is published under the Creative Commons Attribution Noncommercial License, which permits any non-commercial use, distribution, adaptation and reproduction provided the original author(s) and source are given appropriate credit. (C) The Authors 2018

Received: January 22, 2018

Published Online: May 22, 2018

Citation: US Endocrinology. 2018;14(1):27-29

Corresponding Author: Sanjay Kalra, MD, DM, Department of Endocrinology, Bharti Hospital Karnal, India. E: brideknl@gmail.com

Support: No funding was received in the publication of this article.

\section{Diabetes distress}

Diabetes distress (DD) is a well-described challenge of living with diabetes. While earlier authors defined DD as a set of unique, often hidden emotional burdens and worries that are part of the spectrum of patient experience when managing a severe, demanding chronic disease like diabetes, ${ }^{1}$ recent conceptualization views it as an emotional response characterized by extreme apprehension, discomfort or dejection, due to a perceived inability to cope with the challenges and demands of living with diabetes. ${ }^{2}$

DD can be measured using validated scales, including the Diabetes Distress Scale (DDS). ${ }^{3}$ Specific versions of DDS are available for type 1 and type 2 diabetes, as well as parents of teens and partners of adults with type 1 diabetes. These scales help one assess the source of DD. The DDS documents four dimensions of distress. These are: emotional burden, regimen distress, interpersonal distress, and physician distress. ${ }^{4}$ The dimensions of DD in type 1 include powerlessness, management distress, hypoglycemia distress, negative social perceptions, eating distress, physician distress, and friends/ family distress. ${ }^{5}$

\section{Distress and insulin inertia}

Diabetes care providers are well aware of the barriers that prevent timely insulin initiation and intensification. ${ }^{6}$ The bridges that can overcome these barriers are also listed and classified in detail. ${ }^{7}$ The impact of clinical inertia on long-term outcomes is also well known, as are the advantages of timely insulin initiation and intensification. ${ }^{8}$ At times, however, the potential long-term advantages of insulin may be nullified by short-term diabetes "distress." Clinically evident distress in the patient may discourage diabetes care providers from discussing and prescribing biomedically apt therapeutic interventions.

\section{Insulin as a cause of diabetes distress}

One significant contributor to DD is insulin. Even the mere suggestion to initiate insulin is associated with a wide array of emotions among most people. These emotional responses range from innocuous apprehension to significant discomfort and even feelings of dejection, and are caused by a "perceived inability" to cope with the "challenges and demands" of using insulin. Also, it is not uncommon that these emotions are driven by underlying assumptions that are based on myths, rather than facts. However, more importantly, these emotions are real, and cannot be ignored. We therefore suggest that there is a need to address insulin distress as a separate entity. 


\section{Insulin distress}

Insulin distress can be defined as an emotional response to the suggestion to use insulin, characterized by extreme apprehension, discomfort, dejection, or denial, due to a perceived inability to cope with the requirements of insulin therapy.

Insulin distress is a construct based upon the biopsychosocial health model. Persons with diabetes may fear insulin for a variety of reasons. These concerns are perpetuated by a self-sustaining system of hearsay. The availability of easy communication tools, provided by modern technology, have facilitated the spread of such "e-hearsay". 9

\section{Etiopathogenesis}

The etiology of insulin distress may be biomedical, e.g., limited visual, tactile or motor dexterity. It may also be psychosocial in origin, such as fear of social ostracization or lack of financial support.10,11 While insulin distress is usually encountered before or during insulin initiation, it may occur at any time in the course of living with diabetes. Periods of poor glycemic control, poor symptom relief, suggestions for insulin intensification or change in preparation/delivery device may precipitate insulin distress. Distress may also occur in persons who are susceptible to negative reinforcement from friends, relatives, or practitioners of alternative medicine.

\section{Clinical course}

Insulin distress can present with a varied temporal profile and severity. While some persons may have "acute" or short-term distress, which resolves with counseling, others may experience subacute or chronic distress, which is refractory to diabetes education interventions. It is not uncommon to encounter "relapses" of insulin distress, precipitated by illinformed relatives and friends, who counsel against insulin therapy.

\section{Insulin and diabetes distress-distinct entities}

While insulin distress is a part of DD, it is distinct and unique enough to warrant specific consideration. Insulin distress is spread over all domains of DD, as assessed by DDS type 1. Talk about insulin therapy may initially create a sense of powerlessness, and contribute to management distress. concerns about hypoglycemia, negative social perceptions and restriction may be heightened with insulin use. Insulin conversations with physicians and friends/family may also lead to distress due to unfounded concern.

While DD can accompany the lifelong journey of diabetes, insulin distress is usually episodic. Diabetes distress runs a chronic course, with peaks and troughs. On the other hand, insulin distress may be acute, subacute or chronic; monophasic or recurrent. Either may be precipitated by internal events (onset or worsening of complications), by changes in management strategy (frequency and intensity of diagnostic, monitoring, and therapeutic interventions) or by psychosocial factors (suboptimal social or financial environment).

Both of these types of distress respond to non-pharmacological measures, including strengthening of coping skills. While DD requires a generalized approach to management, insulin distress requires more specific and focused interventions.

\section{Disorders of adjustment}

The International Classification of Disease (ICD 10) by the World Health Organization (WHO) states that adjustment disorders are: "states of subjective distress and emotional disturbance, usually interfering with social functioning and performance, and arising in the period of adaptation to a significant life change or to the consequences of a stressful life event (including the presence or possibility of serious physical illness) [...] Individual predisposition or vulnerability plays a greater role in the risk of occurrence and the shaping of the manifestations of adjustment disorders [...] but it is nevertheless assumed that the condition would not have arisen without the stressor. The manifestations vary, and include depressed mood, anxiety, worry (or a mixture of these), a feeling of inability to cope, plan ahead, or continue in the present situation, and some degree of disability in the performance of daily routine". ${ }^{12}$

This clinical picture suggests that insulin distress seems to meet the criteria of an adjustment disorder as specified in ICD $10 .^{12}$

The Diagnostic and Statistical Manual of Mental Disorders (DSM-5) describes adjustment disorder as "the presence of emotional or behavioral symptoms in response to an identifiable stressor(s) occurring within 3 months of the onset of the stressor(s)." In addition to exposure to one or more stressors, other DSM-5 criteria for adjustment disorder must be present:

"The distress is out of proportion with expected reactions to the stressor and/or symptoms must be clinically significant leading to marked distress and impairment in functioning. The distress and impairment should be related to the stressor and are not an escalation of existing mental health disorders. Once the stressor is removed or the person has begun to adjust and cope, the symptoms must subside within six months".

These characteristics also suggest that insulin distress is a disorder of adjustment. However, six months may be an inappropriately long timeframe for assessing the adequacy of adjustment and coping with insulin. ${ }^{13}$

\section{Management}

The management of insulin distress takes significant cues from the management of DD. ${ }^{2}$ It is based upon four cardinal principles, namely, strengthening of self-care skills, optimization of coping skills, minimizing change-related discomfort, and utilization of external support. The umbrella term "coping therapy" can be used to encompass these principles. Insulin distress needs to be managed by addressing patient-related, physicianrelated and drug-related factors.' Strengthening communication and coping skills, along with diabetes/insulin literacy, among all stakeholders, is a prerequisite to creating a sense of comfort with insulin.

The clinical challenge, however, is in deciding whether to address insulin distress before prescribing insulin, or to postpone insulin initiation until distress is resolved (Figure 1).This planning is important, as it will set the stage for further diabetes care, and influence long-term outcomes. An in-depth understanding of both psychology and diabetology is needed to arrive at an appropriate decision.

\section{Take-home messages}

Keeping the aforementioned facts in mind, we suggest the following pragmatic and practical approach to this clinical situation:

1. A thorough biomedical and psychosocial evaluation should precede and accompany discussion regarding insulin in persons with type 2 diabetes. 
2. Diabetes care providers must be aware of insulin distress, its etiology, clinical features, and management.

3. Potential biomedical and psychosocial factors, which may limit insulin acceptance and adherence, must be explored and addressed.

4. The need for insulin, and urgency of insulin prescription, should be balanced with the severity of insulin distress (see Figure 1B).

5. In persons with life-threatening, organ-threatening, or limb-threatening illness for which insulin therapy is needed, insulin distress mitigation strategies should accompany insulin prescription.

6. In persons with low or insignificant insulin distress, insulin therapy should be initiated as per clinical indications. Coping skills should be strengthened in parallel.

7. In persons with significant insulin distress and no immediate threat to health, insulin distress should be addressed prior to starting insulin. Addressing of insulin distress may take one or more clinical interventions.

8. A triage system, as proposed in Figure 1, may help prioritize between insulin initiation and insulin distress mitigation.

9. Comfort level with insulin should be assessed and addressed if necessary, at every clinical encounter in persons using insulin. $\square$
Figure 1: The insulin fulcrum

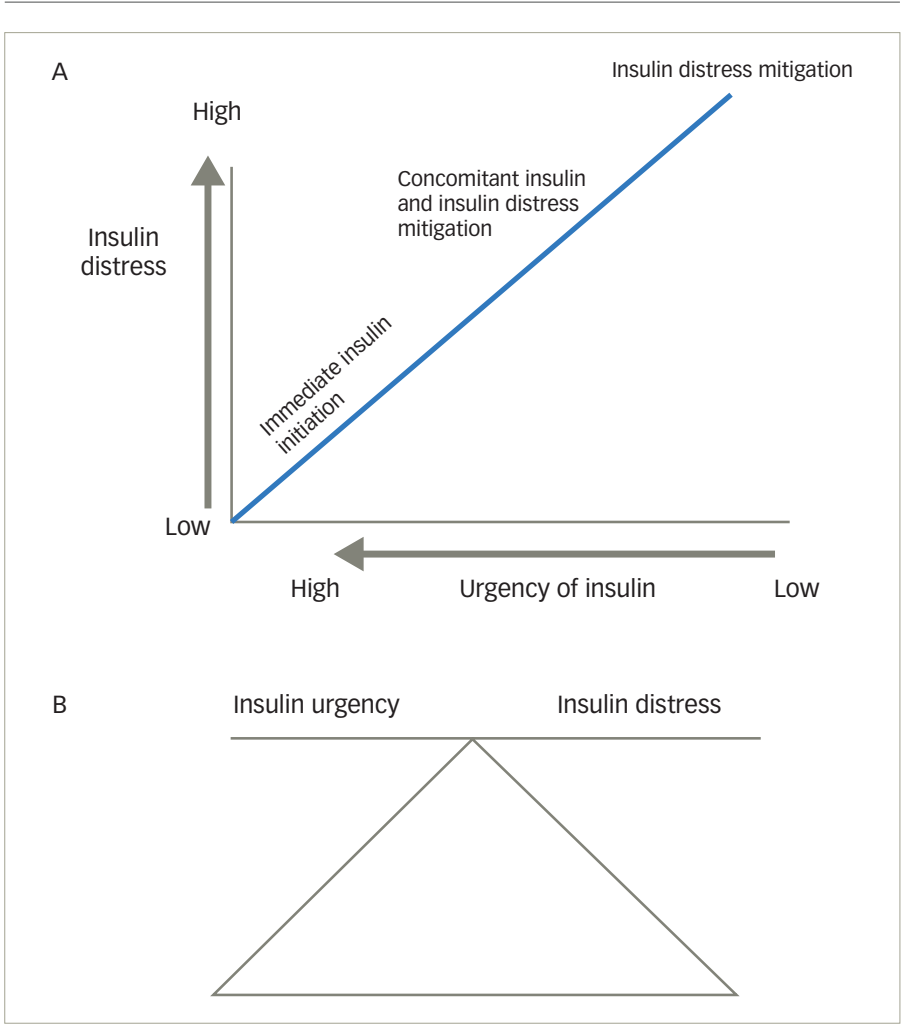

1. Gonzalez JS, Fisher L, Polonsky WH. Depression in diabetes: have we been missing something important? Diabetes Care. 2011;34:236-9

2. Kalra S, Verma K, Singh YB. Management of diabetes distress. Pak Med Assoc. 2017;67:1625-7.

3. Scales and Measures. Available at: http://behavioraldiabetes.org/ scales-and-measures/ (accessed January 1, 2018).

4. Polonsky WH, Fisher L, Earles J, et al. Assessing psychosocial distress in diabetes. Diabetes Care. 2005;28:626-31.

5. Fisher L, Polonsky WH, Hessler DM, et al. Understanding the sources of diabetes distress in adults with type 1 diabetes. $J$
Diabetes Complications. 2015:29:572-7.

6. Kalra S, Gupta Y. Insulin initiation: the triage system. J Pak Med Assoc. 2014;64:1428-30.

7. Kalra S, Ghosal S, Shah P. Consensus on Bridges for Barriers to Insulin Therapy. J Assoc Physicians India. 2017;65:23-30.

8. Khunti K, Millar-Jones D. Clinical inertia to insulin initiation and intensification in the UK: A focused literature review. Prim Care Diabetes. 2017;11:3-12.

9. Kalra S, Balachandran K. De-Hearsay (Diabetes e-Hearsay). J Pak Med Assoc. 2017;67:1293-5

10. Kalra S, Ghosal S. Barriers and bridges to insulin therapy: bio psychosocial classification J Pak Med Assoc. 2017:67:320-1.

11. Tandon N, Kalra S, Balhara YP, et al. Forum for injection technique and therapy expert recommendations, India: The Indian recommendations for best practice in insulin injection technique, 2017. Ind J Endocr Metab, 2017;21:600.

12. World Health Organization. The ICD-10 Classification of Mental and Behavioural Disorders: Clinical Descriptions and Diagnostic Guidelines. Geneva: World Health Organization, 1992.

13. American Psychiatric Association. Diagnostic and Statistical Manual of Mental Disorders (DSM-5 $\left.5^{\oplus}\right)$, Fifth edn. Arlington, US: American Psychiatric Association (APA), 2013. 